\title{
Circuit-Model Analysis of Frequency Selective Surfaces With Scatterers of Arbitrary Geometry
}

\author{
Francisco Mesa, Fellow, IEEE, María García-Vigueras, Francisco Medina, Fellow, IEEE, \\ Raúl Rodríguez-Berral, and Juan R. Mosig, Fellow, IEEE
}

\begin{abstract}
An equivalent-network model is here proposed to characterize two-dimensional planar periodic arrays of arbitrary scatterers/apertures embedded in a layered environment. The model is an extension of the approach previously developed by some of the authors, which only considered simple rectangular scatterers. A key underlying assumption in the present approach is that the current/field distribution in the scatterer can be factorized so that the spatial profile is independent of the frequency in the considered range of interest. This approximation is proven to work properly for a great variety of useful planar scatterer/aperture patterns, even at frequencies within the diffraction regime. The spatial current/field profile is determined from a full-wave simulation at a single and low frequency value. Our numerical results are validated through comparison to commercial simulators for very wide frequency ranges as well as with previously proposed circuit-model approaches.
\end{abstract}

Index Terms-Complex geometries, equivalent circuits, frequency selective surfaces.

\section{INTRODUCTION}

$\mathbf{F}$ REQUENCY selective surfaces (FSS) and other periodic structures are well known as the basis of many useful devices in microwaves and antenna engineering [1], [2]. Thus, a great effort has been devoted to their modeling in order to get efficient tools for analysis and design purposes [3]-[5]. As most of the proposed approaches are of numerical nature, no physical insight about the FSS performance is provided a priori. Therefore, a posteriori eventual explanation of the FSS behavior mostly relies on the operator's skill to adjust appropriately the convenient numerical parameters as well as in his/her ability to find out the relevant physical reasons that explain the obtained numerical output.

Manuscript received May 31, 2014; revised August 13, 2014 and September 01, 2014; accepted September 02, 2014. Date of publication September 10, 2014; date of current version February 02, 2015. This work was supported by the Spanish Ministerio de Ciencia e Innovación and European Union FEDER funds under Projects TEC2010-16948, TEC2013-4193-P, and CSD2008-00066; Junta de Andalucía under Project P12-TIC-1435; and ICT COST Action IC1102.

F. Mesa and R. Rodríguez-Berral are with the Microwaves Group, Department of Applied Physics 1, ETS de Ingeniería Informática, Universidad de Sevilla, 41012 Seville, Spain (e-mail: mesa@us.es; rrberral@us.es).

M. García-Vigueras and J. R. Mosig are with the Laboratory of Electromagnetics and Acoustics, Ecole Polytechnique Federale de Laussane, 1015 Lausanne, Switzerland (e-mail: maria.garciavigueras@epfl.ch).

F. Medina is with the Microwaves Group, Department of Electronics and Electromagnetism, Faculty of Physics, Universidad de Sevilla, 41012 Seville, Spain (e-mail: medina@us.es).

Color versions of one or more of the figures in this letter are available online at http://ieeexplore.iee.org.

Digital Object Identifier 10.1109/LAWP.2014.2356012
Earlier, appropriate equivalent-circuit models [6] were proposed as an insightful alternative to purely numerical methods (the computational cost of numerical methods would have been prohibitive in an early stage of the computer era, and even nowadays still remains a bottleneck). Roughly speaking, most of the reported equivalent networks approaches (ENAs) can be grouped into two categories. The first one encompasses those based on certain restrictive assumptions, such as quasi-static and long-wavelength approximations [7], [8] or homogenization procedures [9]. In such cases, the topology of the network is somehow deduced from a basic theoretical rationale, and the approximate values of the involved parameters are usually given in closed form. Thus, these methods are expected to work properly only within the limited frequency band where the underlying assumptions are satisfied. The second category includes the ENAs whose topology is inferred from the a priori knowledge of the FSS qualitative behavior in the band of interest as well as from some features suggested by the geometry [10], [11]. The specific values of the involved parameters are then extracted from full-wave simulations of the scattering parameters at certain specific frequencies (usually not known in advance). Actually, this approach that combines circuit modeling and a limited use of electromagnetic solvers is becoming a popular trend for the characterization of electromagnetic systems.

Still, a third (and less usual) category of ENAs can be identified in the literature. These approaches use an abinitio methodology to obtain both the appropriate wideband circuit topology and the corresponding closed-form expressions for all the circuit parameters. Relatively few and recent works can be categorized this way (see, for instance, [12]-[14]). Despite their apparent advantages, they suffer from two clear limitations: 1 ) it is implicitly assumed that the spatial profile of the current in the metallic scatterers (or electric field in the apertures) does not change significantly in the considered frequency band; and 2) closed-form expressions can only be provided for canonical geometries of the scatterers (rectangular, circular, etc.).

This letter's goal is to extend the applicability of these last analytical ENAs to deal with noncanonical scatterer/aperture geometries. The price to pay for this generalization is that closedform expressions are no longer available since the current/field in the scatterers/apertures is not analytically known. However, this information can be obtained from a single numerical simulation (performed, for instance, with a commercial software) at a relatively low frequency point. The spatial profile is assumed to be frequency-independent, whereas the frequency-dependent complex amplitude of the surface current/fields is computed fol- 


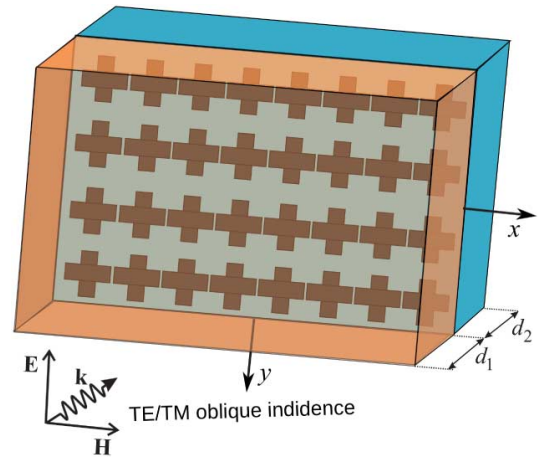

Fig. 1. TE/TM-polarized plane wave impinging on an FSS composed of scatterers of arbitrary geometry that can be embedded/printed in a layered environment. Oblique incidence along principal planes is allowed.

lowing the conceptual scheme provided in [13]-[15]. This approach gives rise to a very efficient tool that combines the advantages of the equivalent-network point of view with the wideapplicability benefits of general-purpose numerical simulators.

\section{EQUiVAlENT Circuit}

The scenario under analysis is shown in Fig. 1; it consists in a time-harmonic (angular frequency $\omega$ ) TE/TM-polarized plane wave that impinges obliquely along the principal plane of an FSS. This FSS is located in the $z=0$ plane, and it is made of scatterers of arbitrary geometry that can be embedded/printed in a layered medium. The unit cell of this periodic structure has dimension $P_{x} \times P_{y}$. For convenience, the case of metallic scatterers will be treated first, leaving the case of aperture-based FSS for a further step. Thus, the current density on the scatterers is assumed to be given by

$$
\mathbf{J}(x, y ; \omega)=F(\omega) \mathbf{J}_{\mathrm{p}}(x, y)
$$

implying that the spatial current profile, $\mathbf{J}_{\mathrm{p}}(x, y)$, is independent of $\omega$. This assumption is key in our approach and has also been the basis of the models derived in [13]-[15]. Although it may seem rather restrictive, this assumption actually covers many relevant practical cases (as proven in Section III).

The current profile of the scatterer at the first resonance has been found to be very similar to the one at low frequencies. Therefore, the previous assumption is expected to be valid up to frequencies below the scatterer first high-order resonance that is compatible with the symmetry conditions imposed by the given excitation. For instance, in the case of dipole-based FSS under normal incidence with the electric field directed along the dipole, this approximation is found valid up to frequencies close to and below the third intrinsic resonance of the metallic dipole (the spatial profile of the current in the first resonance $\left[\lambda \sim 2 l_{\text {dip }}\right]$ is very similar to the quasi-static spatial profile; the second resonance $\left[\lambda \sim l_{\mathrm{dip}}\right]$ is not compatible with the excitation; only close to the third resonance $\left[\lambda \sim 2 / 3 l_{\text {dip }}\right]$, the quasi-static profile fails to match the spatial variations imposed by the resonance condition). For complex geometries, it is not so simple to find an estimation of the frequency range of validity as the resonance conditions are not readily known. For instance, in the case of metallic rings under normal incidence, the expected validity range should extend, at maximum, up to frequencies where the

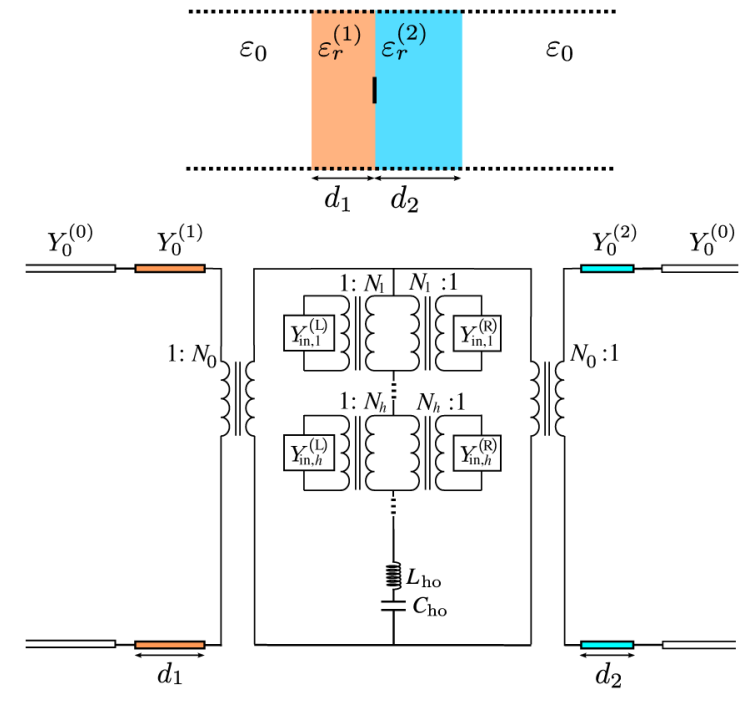

Fig. 2. Topology of the equivalent circuit for path-based FSS. This equivalent circuit is a modified version of the circuits in [13] and [14].

average perimeter of the ring, $P_{\mathrm{av}}$, satisfies $P_{\mathrm{av}} \sim 3 \lambda$ (under oblique incidence, the condition is $P_{\mathrm{av}} \sim 2 \lambda$ ).

Following a Floquet's analysis similar to that reported in [13] and [14], it can be proven that the reflection coefficient of the obliquely incident plane wave can be written as

$$
R=\frac{Y_{\mathrm{in}, 0}^{\mathrm{L}} /\left|N_{0}\right|^{2}-Y_{\mathrm{in}, 0}^{\mathrm{R}} /\left|N_{0}\right|^{2}-Z_{\mathrm{eq}}^{-1}}{Y_{\mathrm{in}, 0}^{\mathrm{L}} /\left|N_{0}\right|^{2}+Y_{\mathrm{in}, 0}^{\mathrm{R}} /\left|N_{0}\right|^{2}+Z_{\mathrm{eq}}^{-1}}
$$

where

$$
Z_{\mathrm{eq}}=\frac{1}{\mathrm{j} \omega C_{\mathrm{ho}}}+\mathrm{j} \omega L_{\mathrm{ho}}+\sum_{h=-M}^{M} \frac{\left|N_{h}\right|^{2}}{Y_{\mathrm{in}, h}^{(\mathrm{L})}+Y_{\mathrm{in}, h}^{(\mathrm{R})}}
$$

and whose associated circuit topology is depicted in Fig. 2. Looking at the figure, the index $h$ is a proxy for any of the different $n m$ harmonics with wave vector $\mathbf{k}_{\mathrm{t}, h}$ that comprise the Floquet expansion of the field $[h=0$ stands for the incident TE/TM uniform plane wave and is excluded in (3)]. This wave vector and related variables are given by

$$
\begin{aligned}
\mathbf{k}_{\mathrm{t}, h} & =k_{x n} \hat{\mathbf{x}}+k_{y m} \hat{\mathbf{y}} \\
& =\left(k_{x 0}+k_{n}\right) \hat{\mathbf{x}}+\left(k_{y 0}+k_{m}\right) \hat{\mathbf{y}} \\
\hat{\mathbf{t}}_{h} & =\frac{\mathbf{k}_{\mathrm{t}, h}}{\left|\mathbf{k}_{\mathbf{t}, h}\right|}=\frac{k_{x n} \hat{\mathbf{x}}+k_{y m} \hat{\mathbf{y}}}{\sqrt{k_{x n}^{2}+k_{y m}^{2}}} \\
\hat{\mathbf{u}}_{h} & =\hat{\mathbf{z}} \times \hat{\mathbf{t}}_{h}=\frac{k_{x n} \hat{\mathbf{y}}-k_{y m} \hat{\mathbf{x}}}{\sqrt{k_{x n}^{2}+k_{y m}^{2}}} \\
\hat{\mathbf{v}}_{h} & =\left\{\begin{array}{cc}
\hat{\mathbf{t}}_{h}, & \text { TM harmonics } \\
-\hat{\mathbf{u}}_{h}, & \text { TE harmonics }
\end{array}\right.
\end{aligned}
$$

with $k_{x 0}=k_{0} \sin \theta \cos \varphi$ and $k_{y 0}=k_{0} \sin \theta \sin \varphi$ being the transverse wavevector components of the incident field ( $\varphi=0, \pi / 2$, since only principal-plane incidence is considered) and $k_{n}=2 \pi n / P_{x}, k_{m}=2 \pi m / P_{y}\left(k_{0}\right.$ is the vacuum wavenumber). $Y_{\mathrm{in}, h}^{(\mathrm{L})}$ and $Y_{\mathrm{in}, h}^{(\mathrm{R})}$ represent the corresponding input admittances to the left/right (L/R) cascade of dielectrics whose wave admittances $Y_{h}^{(i)}$ are given by

$$
Y_{h}^{(i)}=\frac{H_{\mathrm{t}, h}}{E_{\mathrm{t}, h}}=\frac{1}{\eta^{(i)}} \begin{cases}k^{(i)} / \beta_{n m}^{(i)}, & \text { TM harmonics } \\ \beta_{n m}^{(i)} / k^{(i)}, & \text { TE harmonics }\end{cases}
$$


where $k^{(i)}=\sqrt{\varepsilon_{r}^{(i)}} k_{0}, \beta_{h}^{(i)}=\sqrt{\varepsilon_{r}^{(i)} k_{0}^{2}-\left|\mathbf{k}_{\mathrm{t}, h}\right|^{2}}$, and $\eta^{(i)}=\eta_{0} / \sqrt{\varepsilon_{r}^{(i)}}$, with $\eta_{0}$ being the impedance of free space. The $N_{h}$ coefficients in (2) and (3) represent transformer ratios (see Fig. 2) and are given by

$$
N_{h}=\tilde{\mathbf{J}}_{\mathrm{p}}^{*}\left(\mathbf{k}_{\mathrm{t}, h}\right) \cdot \hat{\mathbf{v}}_{h}
$$

where symbol “ ” stands for Fourier transform with respect to $x, y$. The factors that weighted the impedances in our previous works [13], [14] have been represented here as transformer turn ratios following our recent work in [15]. Details about the meaning of $C_{\mathrm{ho}}$ and $L_{\mathrm{ho}}$ (frequency-independent capacitance and inductance associated with high-order evanescent harmonics) can be found in [13] and [14].

Now it should be considered that $\tilde{\mathbf{J}}_{\mathrm{p}}\left(\mathbf{k}_{\mathrm{t}, h}\right)$ is only known in closed form for very specific and canonical geometries [16]. However, for any other geometry, $\tilde{\mathbf{J}}_{\mathrm{p}}\left(\mathbf{k}_{\mathrm{t}, h}\right)$ can be numerically constructed from the $\mathbf{J}_{\mathrm{p}}(x, y)$ provided by any full-wave electromagnetic solver. Here, HFSS [17] has been employed for this purpose, with $\mathbf{J}_{\mathrm{p}}(x, y)$ being extracted from a single and low-frequency simulation of the free-standing periodic array (if these full-wave results are affected by numerical noise, the use of a convenient filter is advisable). Next, the fast Fourier transform (FFT) algorithm is used to obtain $\tilde{\mathbf{J}}_{\mathrm{p}}\left(\mathbf{k}_{\mathrm{t}, h}\right)$, which is introduced in (9) to directly compute the frequency-independent $N_{h}$ coefficients. (The following functions from the python open-source library SciPy [18] have been used in this work: griddata to create a regular grid from the nonuniform mesh data provided by the simulator, fft2 to compute the 2-D Fast Fourier Transform, savitzky_golay to filter the noisy data. Nevertheless, other convenient specific commercial or opensource software could be found for the required computational tasks.) The remaining frequency-dependent parameters of the circuit are known in closed form.

As it is explained in [13] and [14], aperture-based FSS can be treated analogously, and the circuit elements associated to the discontinuity should be shunt connected. The only relevant aspect to be considered in this case is that the computation of the required spatial profile of the field in the aperture, $\mathbf{E}_{\mathrm{a}}(x, y)$, can directly be obtained from the current profile of the dual geometry (Babinet's principle is valid for free-standing FSS). The current profile $\mathbf{J}_{\mathrm{p}}(x, y)$ can be employed to obtain the aperture field in the dual structure in the following way: $\mathbf{E}_{\mathrm{a}}(x, y)=$ $\hat{\mathbf{z}} \times \mathbf{J}_{\mathrm{p}}(x, y)$.

\section{RESULTS}

Next, our approach will be validated by comparing our circuit-model results to other data previously published in the literature. The first structure to be analyzed is a crossed-dipole FSS printed on a dielectric slab, which has frequently been used in the past as benchmark. Our results are compared in Fig. 3 to both the method-of-moment (MoM) results provided in [5, Fig. 9] and HFSS data. Very good agreement is observed with [5] in the frequency range considered in that paper. The comparison to HFSS simulations in a more extended range shows that our approach gives good results over a considerably wide frequency band. Once we have the spatial profile of the current provided

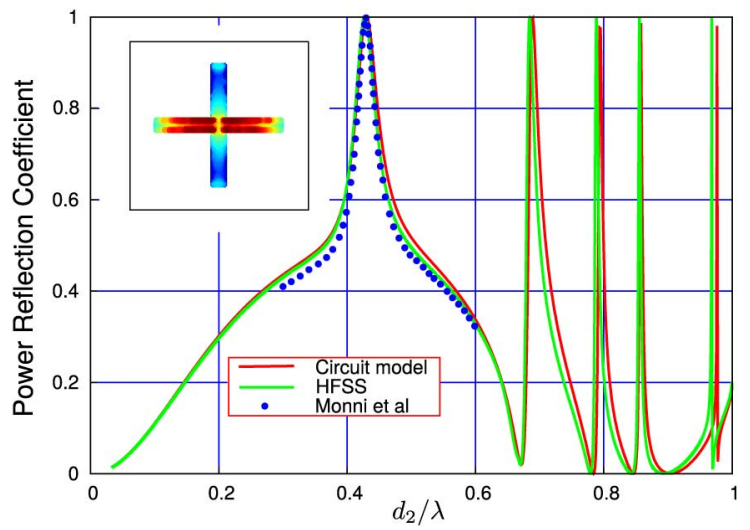

Fig. 3. Power reflection coefficient of a crossed-dipole FSS printed on a dielectric slab with $d_{2}=3 \mathrm{~mm}, \varepsilon_{\mathrm{r}}^{(2)}=4.0\left(d_{1}=0\right)$. Comparison to the results reported in [5, Fig. 9] and HFSS. $P_{x}=P_{y}=10 \mathrm{~mm}$. The dimension of the metallic crossed dipole is given in [5, Fig. 9]. The inset shows the numerical current pattern.

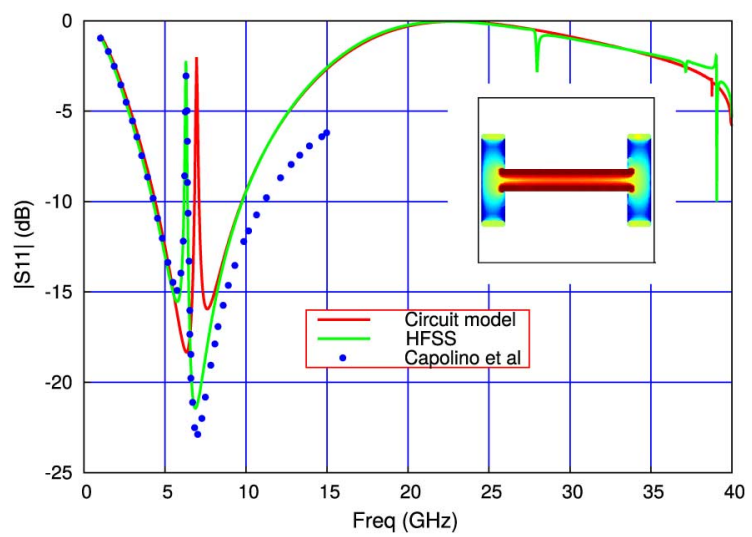

Fig. 4. Transmission coefficient for a layer of dogbone pairs separated by a dielectric spacer of thickness $d=0.8 \mathrm{~mm}, \varepsilon_{\mathrm{r}}=3.48$, and $\tan \delta=0.004$. $P_{x}=P_{y}=7.51 \mathrm{~mm}$. The employed current pattern is shown in the inset.

by HFSS at a single low-frequency value (2 GHz), the CPU time employed by our approach to compute the 1000 frequency points used in Fig. 3 is almost negligible (a few seconds in a typical laptop) in comparison to the total time required by HFSS to cover this wide band.

In Fig. 4, we consider the layer of coupled dogbone pairs studied in [11, Fig. 2]. The authors of that paper also provide an equivalent $\mathrm{X}$-shaped lumped circuit model for the metalayer formed by arrayed pairs of dogbone-shaped conductors printed on the two sides of a dielectric substrate. The parameters of that equivalent network are chosen to match the resonances of the structure, which implies that the full-wave behavior of the structure must be known in advance. As explained in Section I, no a priori information is needed to build our circuit model, and the results for this coupled structure are obtained after applying the well-known theory of odd/even excitations (electric/magnetic wall at the middle plane of the substrate). These results are in acceptable agreement with those in [11] within the frequency band considered in that work. Moreover, Fig. 4 also shows that our results are in good agreement with HFSS data in a much wider frequency band. Beyond $27 \mathrm{GHz}$, disagreements with HFSS appear as the spatial profile of the current does no longer remain constant at such frequencies. 


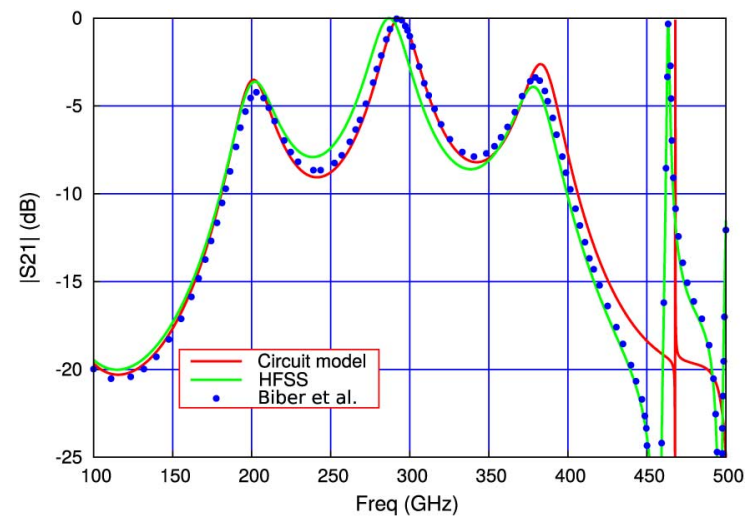

Fig. 5. Frequency response of the $300-\mathrm{GHz}$ passband filter 3D1 with dogbone-shaped holes proposed in [4, Fig. 2] under oblique incidence $\left(\theta=20^{0}\right.$, $\left.\varphi=90^{\circ}\right)$. The perforated metallic screen is printed on a silicon layer with $h=$ $302 \mu \mathrm{m}$ and $\varepsilon_{\mathrm{r}}=11.8$. Dimensions of the scatterer are given in [4, Fig. 8].

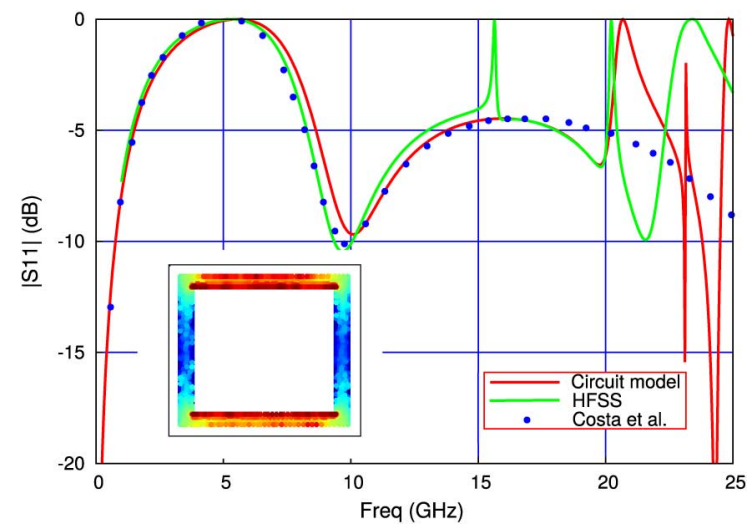

Fig. 6. Reflection coefficient of a squared-ring FSS sandwiched between two dielectric slabs $\left(d_{1}=1 \mathrm{~mm}, \varepsilon_{\mathrm{r}}^{(1)}=3.0, d_{2}=2 \mathrm{~mm}, \varepsilon_{\mathrm{r}}^{(2)}=5.0\right)$ under normal incidence. Comparison to the results reported in [10, Fig. 15] and HFSS. $P_{x}=P_{y}=10 \mathrm{~mm}$. The dimensions of the metallic square ring are given in [10, Fig. 15]. The employed current pattern is sketched in the inset.

The case considered in Fig. 5 corresponds to the THz passband filter made with dogbone-shaped holes previously studied in [4, Fig. 8]. The good agreement between our circuit-model data and both the full-wave results provided in [4] and HFSS shows that our approach also deals efficiently with aperturebased FSSs. As mentioned before, the aperture field is obtained from the current on the dual scatterer.

Next, we present a final example that clearly illustrates the frequency range of validity of our model. The squared-ring FSS treated in [10, Fig. 15] is now analyzed in Fig. 6. The scatterer of this FSS allows for large electric current paths that give place to high-order resonances before the grating-lobe regime.

It can be observed that our results show important disagreements with HFSS beyond $15 \mathrm{GHz}$. As explained in Section II, this result could be expected since, at about $15 \mathrm{GHz}$, the perimeter of the square ring approaches three wavelengths (the resonance associated with $P_{\mathrm{av}} \sim 2 \lambda$ is inhibited under the given normal-incidence conditions). Certainly, at these high frequencies, the current does not match the profile found at low frequencies although, for certain range up to $20 \mathrm{GHz}$, our results still present an acceptable agreement without the need of the complete full-wave behavior in advance as in [10].

\section{CONCLUSION}

In this letter, we have improved significantly the practical applicability of previous circuit models developed by some of the authors. With limited assistance from a full-wave simulator, the present extended version gives rise not only to an efficient analysis/design tool, but also a very convenient, physically insightful circuit model.

\section{REFERENCES}

[1] D. Sievenpiper, L. Zhang, R. F. J. Broas, N. G. Alexópoulos, and E. Yablonovitch, "High-impedance electromagnetic surfaces with a forbidden frequency band," IEEE Trans. Microw. Theory Tech., vol. 47, no. 11, pp. 2059-2074, Nov. 1999.

[2] B. Munk, Frequency Selective Surfaces: Theory and Design. New York, NY, USA: Wiley, 2000.

[3] S. Maci, M. Caiazzo, A. Cucini, and M. Casaletti, "A pole-zero matching method for EBG surfaces composed of a dipole FSS printed on a grounded dielectric slab," IEEE Trans. Antennas Propag., vol. 53, no. 1, pp. 70-81, Jan. 2005.

[4] S. Biber, M. Bozzi, O. Günther, L. Perregrini, and L.-P. Schmidt, "Design and testing of frequency-selective surfaces on silicon substrates for submillimeter-wave applications," IEEE Trans. Antennas Propag., vol. 54, no. 9, pp. 2638-2645, Sep. 2006.

[5] S. Monni, G. Gerini, A. Neto, and A. G. Tijhuis, "Multi-mode equivalent networks for the design and analysis of frequency selective surfaces," IEEE Trans. Antennas Propag., vol. 55, no. 10, pp. 2824-2835, Oct. 2007.

[6] L. B. Felsen and A. A. Oliner, "Determination of equivalent circuit parameters for dissipative microwave structures," Proc. IRE, vol. 42, no. 2, pp. 477-483, Feb. 1954.

[7] S. A. Tretyakov, Analytical Modeling in Applied Electromagnetics. Norwood, MA, USA: Artech House, 2003.

[8] C. S. R. Kaipa, A. B. Yakovlev, F. Medina, and F. Mesa, "Transmission through stacked 2-D periodic distributions of square conducting patches," J. Appl. Phys., vol. 112, p. 033101, Aug. 2012.

[9] O. Luukkonen et al., "Simple and accurate analytical model of planar grids and high-impedance surfaces comprising metal strips or patches," IEEE Trans. Antennas Propag., vol. 56, no. 6, pp. 1624-1632, Jun. 2008.

[10] F. Costa, A. Monorchio, and G. Manara, "Efficient analysis of frequency-selective surfaces by a simple equivalent-circuit model," IEEE Antennas Propag. Mag., vol. 54, no. 4, pp. 35-48, Aug. 2012.

[11] F. Capolino, A. Vallecchi, and M. Albani, "Equivalent transmission line model with a lumped X-circuit for a metalayer made of pairs of planar conductors," IEEE Trans. Antennas Propag., vol. 61, no. 2, pp. 852-861, Feb. 2013.

[12] R. Dubrovka, J. Vazquez, C. Parini, and D. Moore, "Equivalent circuit method for analysis and synthesis of frequency selective surfaces," IEE Proc. Pt. H, Microw. Antennas Propag., vol. 153, no. 3, pp. 213-220, Mar. 2006.

[13] R. Rodríguez-Berral, C. Molero, F. Medina, and F. Mesa, "Analytical wideband model for strip/slit gratings loaded with dielectric slabs," IEEE Trans. Microw. Theory Tech., vol. 60, no. 12, pp. 3908-3918, Dec. 2012.

[14] R. Rodríguez-Berral, F. Mesa, F. Medina, and M. García-Vigueras, "Analytical circuit model for dipole frequency-selective surfaces," in Proc. IEEE IMS, Seattle, WA, USA, Jun. 2013, pp. 1-4.

[15] R. Rodriguez-Berral, F. Mesa, and F. Medina, "Circuit model for patch/slot-based frequency selective surfaces under conical incidence," in Proc. EuCAP, The Hague, The Netherlands, Apr. 2014, pp. 1714-1718.

[16] S. R. Rengarajan, "Choice of basis functions for accurate characterization of infinite array of microstrip reflectarray elements," IEEE Antennas Wireless Propag. Lett., vol. 4, pp. 47-50, 2005.

[17] High Frequency Structure Simulator (HFSS). ANSYS, Canonsburg, PA, USA, 2013 [Online]. Available: http://www.ansys.com/Products/ Simulation+Technology/Electromagnetics/High-Performance+Electronic+Design/ANSYS+HFSS

[18] SciPy, "Open source scientific tools for Python," [Online]. Available: http://www.scipy.org 